\title{
SHARP WEIGHTED ESTIMATES FOR APPROXIMATING DYADIC OPERATORS
}

\author{
DAVID CRUZ-URIBE, SFO, JOSÉ MARÍA MARTELL, AND CARLOS PÉREZ
}

Abstract. We give a new proof of the sharp weighted $L^{p}$ inequality

$$
\|T\|_{L^{p}(w)} \leq C_{n, T}[w]_{A_{p}}^{\max \left(1, \frac{1}{p-1}\right)},
$$

where $T$ is the Hilbert transform, a Riesz transform, the BeurlingAhlfors operator or any operator that can be approximated by Haar shift operators. Our proof avoids the Bellman function technique and two weight norm inequalities. We use instead a recent result due to A. Lerner [15] to estimate the oscillation of dyadic operators.

The method we use is flexible enough to obtain the sharp oneweight result for other important operators as well as a very sharp two-weight bump type result for $T$ as can be found in [5].

\section{INTRODUCTION}

One weight norm inequalities for singular integrals of the form

$$
\|T f\|_{L^{p}(w)} \leq C\|f\|_{L^{p}(w)} \quad w \in A_{p},
$$

have a long history, beginning with the work of Hunt, Muckenhoupt and Wheeden [12] for the Hilbert transform. (See Duoandikoetxea [7] for a concise history.) The constant $C$ depends on the $A_{p}$ constant of the weight $w$ :

$$
[w]_{A_{p}}=\sup _{Q} f_{Q} w(x) d x\left(f_{Q} w(x)^{1-p^{\prime}} d x\right)^{p-1}
$$

Date: January 25, 2010.

1991 Mathematics Subject Classification. 42B20, 42B25.

Key words and phrases. $A_{p}$ weights, Haar shift operators singular integral operators, Hilbert transform, Riesz transforms, Beurling-Ahlfors operator, dyadic square function, vector-valued maximal operator.

The first author was supported by a grant from the Faculty Research Committee and the Stewart-Dorwart Faculty Development Fund at Trinity College; the first and third authors are supported by grant MTM2009-08934 from the Spanish Ministry of Science and Innovation; the second author is supported by grant MTM2007-60952 from the same institution and by CSIC PIE 200850I015. 
An interesting question is the exact dependence on the $A_{p}$ constant. This was first investigated by Buckley [2]. More recently, this problem has attracted renewed attention because of the work of Astala, Iwaniec and Saksman [1]. They proved sharp regularity results for solutions to the Beltrami equation, assuming that the operator norm of the Beurling-Ahlfors transform growths linearly in terms of the $A_{2}$ constant. This was proved by S. Petermichl and A. Volberg [20] and by Petermichl $[18,19]$ for the Hilbert transform and the Riesz Transforms. In these papers it has been shown that if $T$ is any of these operators, then

$$
\|T\|_{L^{p}(w)} \leq c_{p, n}[w]_{A_{p}}^{\max \left(1, \frac{1}{p-1}\right)} \quad 1<p<\infty
$$

and the exponent $\max \left(1, \frac{1}{p-1}\right)$ is best possible. It has been conjectured that the same estimate holds for any Calderón-Zygmund operator $T$. By the sharp version of the Rubio de Francia extrapolation theorem due to Dragičević et al. [6], it suffices to prove this inequality for $p=2$, namely

$$
\|T\|_{L^{2}(w)} \leq c_{n}[w]_{A_{2}} .
$$

In each of the known cases, the proof used a technique developed by Petermichl [17] to reduce the problem to proving the analogous inequality for a corresponding Haar shift operator. The norm inequalities for these dyadic operators were then proved using Bellman function techniques.

Recently, Lacey, Petermichl and Reguera-Rodriguez [14] gave a proof of the sharp $A_{2}$ constant for a large family of Haar shift operators that includes all of the dyadic operators needed for the above results. Their proof avoids the use of Bellman functions, and instead uses a deep, twoweight " $T b$ theorem" for Haar shift operators due to Nazarov, Treil and Volberg [16].

We give a different proof that avoids both Bellman functions and two-weight norm inequalities such as the $T b$ theorem. Instead, we use a very interesting decomposition argument using local mean oscillation recently developed by Lerner [15].

An important advantage of our approach is that it also yields the optimal sharp one weight norm inequalities for other operators such as dyadic square functions and paraproducts, maximal singular integrals and the vector-valued maximal function of C. Fefferman-Stein. Moreover it also gives very sharp two weight " $A_{p}$ bump" type conditions that improve results gotten by the authors in [4]. All these results can be found in [5]. Key to our approach is that the operators are either dyadic or can be approximated by dyadic operators (e.g., by the Haar 
shift operators defined below). Thus all these results will extend to any operator that can be approximated in this way.

To state our result we first give some definitions following [14] and consider simultaneously a family of dyadic operators - the Haar shift operators - that contains all the operators we are interested in.

Let $\Delta$ be the set of dyadic cubes in $\mathbb{R}^{n}$. For our arguments we properly need to consider the sets $\Delta_{s, t}, s \in \mathbb{R}^{n}, t>0$, of translations and dilations of dyadic cubes. However, it will be immediate that all of our arguments for dyadic cubes extend to these more general families, so without loss of generality we will restrict ourselves to dyadic cubes.

We define a Haar function on a cube $Q \in \Delta$ to be a function $h_{Q}$ such that

(a) $\operatorname{supp}\left(h_{Q}\right) \subset Q$;

(b) if $Q^{\prime} \in \Delta$ and $Q^{\prime} \subsetneq Q$, then $h_{Q}$ is constant on $Q^{\prime}$;

(c) $\left\|h_{Q}\right\|_{\infty} \leq|Q|^{-1 / 2}$

(d) $\int_{Q} h_{Q}(x) d x=0$.

Given an integer $\tau \geq 0$, a Haar shift operator of index $\tau$ is an operator of the form

$$
H_{\tau} f(x)=\sum_{Q \in \Delta} \sum_{\substack{Q^{\prime}, Q^{\prime \prime} \in \Delta(Q) \\ 2^{-\tau n}|Q| \leq\left|Q^{\prime}\right|,\left|Q^{\prime \prime}\right|}} a_{Q^{\prime}, Q^{\prime \prime}}\left\langle f, h_{Q^{\prime}}\right\rangle h_{Q^{\prime \prime}}(x),
$$

where $a_{Q^{\prime}, Q^{\prime \prime}}$ is a constant such that

$$
\left|a_{Q^{\prime}, Q^{\prime \prime}}\right| \leq C\left(\frac{\left|Q^{\prime}\right|}{|Q|} \frac{\left|Q^{\prime \prime}\right|}{|Q|}\right)^{1 / 2}
$$

We say that $H_{\tau}$ is a CZ Haar shift operator if it is bounded on $L^{2}$.

An important example of a Haar shift operator when $n=1$ is the Haar shift (also known as the dyadic Hilbert transform) $H^{d}$, defined by

$$
H^{d} f(x)=\sum_{I \in \Delta}\left\langle f, h_{I}\right\rangle\left(h_{I_{-}}(x)-h_{I_{+}}(x)\right),
$$

where, as before, given a dyadic interval $I, I_{+}$and $I_{-}$are its right and left halves, and

$$
h_{I}(x)=|I|^{-1 / 2}\left(\chi_{I_{-}}(x)-\chi_{I_{+}}(x)\right) .
$$

Clearly $h_{I}$ is a Haar function on $I$ and one can write $H^{d}$ as a Haar shift operator of index $\tau=1$ with $a_{I^{\prime}, I^{\prime \prime}}= \pm 1$ for $I^{\prime}=I, I^{\prime \prime}=I_{ \pm}$ and $a_{I^{\prime}, I^{\prime \prime}}=0$ otherwise. These are the operators used by Petermichl $[17,18]$ to approximate the Hilbert transform. More precisely, she used the family of operators $H_{s, t}^{d}, s \in \mathbb{R}, t>0$, which are defined as above but with the dyadic grid replaced by its translation by $s$ and 
dilation by $t$. The Hilbert transform is then the limit of integral averages of these operators, so norm inequalities for $H$ follow from norm inequalities for $H_{s, t}^{d}$ by Fatou's lemma and Minkowski's inequality. Similar approximations hold for the Riesz transforms and Beurling-Ahlfors operator, and we refer the reader to $[19,20]$ for more details.

We can now state our main result.

Theorem 1. Let $H_{\tau}$ be a CZ Haar shift operator where $\tau \geq 0$ is an integer. Then for every $w \in A_{2}$,

$$
\left\|H_{\tau}\right\|_{L^{2}(w)} \leq C(\tau, n)[w]_{A_{2}} .
$$

As a consequence, the same norm inequality holds for the Hilbert transform, the Riesz transforms, and the Beurling-Ahlfors operator.

If we apply the sharp version of the Rubio de Francia extrapolation theorem [6] mentioned above, we get sharp $L^{p}$ estimates.

Corollary 2. Let $H_{\tau}$ as above and let $1<p<\infty$. Then for every $w \in A_{p}$,

$$
\left\|H_{\tau}\right\|_{L^{p}(w)} \leq C(\tau, n, p)[w]_{A_{p}}^{\max \left(1, \frac{1}{p-1}\right)} .
$$

As a consequence, the same norm inequality holds for the Hilbert transform, the Riesz transforms, and the Beurling-Ahlfors operator.

The remainder of this paper is organized as follows. In Section 2 we give some preliminary definitions and state Lerner's decomposition theorem. In Section 3 we prove an estimate which allows us to apply this decomposition to the CZ Haar shift operators. In Section 4 we prove Theorem 1.

\section{LOCAL MEAN OSCILLATiON}

We begin with some basic definitions. We follow the terminology and notation of Lerner [15], which in turn is based on Fujii [8, 9] and Jawerth and Torchinsky [13]. We note in passing that many of the underlying ideas originated in the work of Carleson [3] and Garnett and Jones [10].

Hereafter we assume that all functions $f$ are measurable and finitevalued almost everywhere. Given a cube $Q$ and $\lambda, 0<\lambda<1$, define the local mean oscillation of $f$ on $Q$ by

$$
\omega_{\lambda}(f, Q)=\inf _{c \in \mathbb{R}}\left((f-c) \chi_{Q}\right)^{*}(\lambda|Q|),
$$


where $f^{*}$ is the non-increasing rearrangement of $f$. The local sharp maximal function of $f$ relative to $Q$ is then defined by

$$
M_{\lambda, Q}^{\#} f(x)=\sup _{\substack{Q^{\prime} \ni x \\ Q^{\prime} \subset Q}} \omega_{\lambda}(f, Q) .
$$

A median value of $f$ on $Q$ is a (possibly not unique) number $m_{f}(Q)$ such that both

$$
\begin{aligned}
& \left|\left\{x \in Q: f(x)>m_{f}(Q)\right\}\right| \leq \frac{|Q|}{2} \\
& \left|\left\{x \in Q: f(x)<m_{f}(Q)\right\}\right| \leq \frac{|Q|}{2} .
\end{aligned}
$$

The median plays the same role for the local sharp maximal function as the mean does for the C. Fefferman-Stein sharp maximal function. More precisely, for each $\lambda, 0<\lambda \leq 1 / 2$,

$$
\omega_{\lambda}(f, Q) \leq\left(\left(f-m_{f}(Q)\right) \chi_{Q}\right)^{*}(\lambda|Q|) \leq 2 \omega_{\lambda}(f, Q) .
$$

To estimate the median and the local mean oscillation we need the following properties that follow from the definition of rearrangements. For any function $f, \lambda, 0<\lambda<1, p, 0<p<\infty$, and cube $Q$,

$$
\begin{aligned}
& \left(f \chi_{Q}\right)^{*}(\lambda|Q|) \leq \lambda^{-1 / p}\|f\|_{L^{p, \infty}\left(Q,|Q|^{-1} d x\right)}, \\
& \left(f \chi_{Q}\right)^{*}(\lambda|Q|) \leq\left(\frac{1}{\lambda|Q|} \int_{Q}|f|^{p} d x\right)^{1 / p} .
\end{aligned}
$$

Inequality (3) is central to our proofs as it allows us to use weak $(1,1)$ inequalities directly in our estimates. By way of comparison, in [4] a key technical difficulty resulted from having to use Kolmogorov's inequality rather than the weak $(1,1)$ inequality for a singular integral. Overcoming this is the reason the results there were limited to log bumps.

Finally, from the definition of rearrangements we have that

$$
\left|m_{f}(Q)\right| \leq\left(f \chi_{Q}\right)^{*}(|Q| / 2),
$$

and so by (4), if $f \in L^{p}$ for any $p>0$, then $m_{f}(Q) \rightarrow 0$ as $|Q| \rightarrow \infty$.

Finally, to state Lerner's decomposition theorem, we use the following notation. Given a cube $Q_{0}$, let $\Delta\left(Q_{0}\right)$ be the collection of dyadic cubes relative to $Q_{0}$. And given $Q \in \Delta\left(Q_{0}\right), Q \neq Q_{0}$, let $\widehat{Q}$ be its dyadic parent: the unique dyadic cube containing $Q$ whose side-length is twice that of $Q$. 
Theorem 3. ([15]) Given a measurable function $f$ and a cube $Q_{0}$, for each $k \geq 1$ there exists a (possibly empty) collection of pairwise disjoint cubes $\left\{Q_{j}^{k}\right\} \subset \Delta\left(Q_{0}\right)$ such that if $\Omega_{k}=\bigcup_{j} Q_{j}^{k}$, then $\Omega_{k+1} \subset \Omega_{k}$ and $\left|\Omega_{k+1} \cap Q_{j}^{k}\right| \leq \frac{1}{2}\left|Q_{j}^{k}\right|$. Furthermore, for almost every $x \in Q_{0}$,

$$
\left|f(x)-m_{f}\left(Q_{0}\right)\right| \leq 4 M_{\frac{1}{4}, Q_{0}}^{\#} f(x)+4 \sum_{k, j} \omega_{\frac{1}{2^{n+2}}}\left(f, \widehat{Q}_{j}^{k}\right) \chi_{Q_{j}^{k}}(x) .
$$

Remark 4. If for all $j$ and $k$ we define $E_{j}^{k}=Q_{j}^{k} \backslash \Omega_{k+1}$, then the sets $E_{j}^{k}$ are pairwise disjoint and $\left|E_{j}^{k}\right| \geq \frac{1}{2}\left|Q_{j}^{k}\right|$.

Remark 5. Though it is not explicit in [15], it follows at once from the proof that we can replace $M_{\frac{1}{4}, Q_{0}}^{\#}$ by the corresponding dyadic operator $M_{\frac{1}{4}, Q_{0}}^{\#, d}$, where

$$
M_{\lambda, Q}^{\#, d} f(x)=\sup _{x \in Q^{\prime} \in \Delta(Q)} \omega_{\lambda}\left(f, Q^{\prime}\right) .
$$

Intuitively, one may think of the cubes $\left\{Q_{j}^{k}\right\}$ as being the analog of the Calderón-Zygmund cubes for the function $f-m_{f}\left(Q_{0}\right)$ but defined with respect to the median instead of the mean.

\section{LOCAL MEAN oscillation of the HaAR SHIFT Operators}

To apply Theorem 3 to the Haar shift operators we need two lemmas. The first is simply that CZ Haar shift operators satisfy a weak $(1,1)$ inequality. The proof of this is known but we could not find it in the literature and it is explicit in [5]. Here and below we will use the following notation: given an integer $\tau \geq 0$ and a dyadic cube $Q$, let $Q^{\tau}$ denote its $\tau$-th generation "ancestor": that is, the unique dyadic cube $Q^{\tau}$ containing $Q$ such that $\left|Q^{\tau}\right|=2^{\tau n}|Q|$.

Lemma 6. Given an integer $\tau \geq 0$, there exists a constant $C_{\tau, n}$ such that for every $t>0$,

$$
\left\|H_{\tau} f\right\|_{L^{1, \infty}\left(\mathbb{R}^{n}\right)} \leq C_{\tau, n} \int_{\mathbb{R}^{n}}|f(x)| d x .
$$

Our second lemma is a key estimate that is sharper variant of a result known for Calderón-Zygmund singular integrals (see [13]) and whose proof is similar. For completeness we include the details.

Lemma 7. Given $\tau \geq 0$, let $H_{\tau}$ be a CZ Haar shift operator. Fix $\lambda$, $0<\lambda \leq 1 / 2$. Then for any function $f$, every dyadic cube $Q_{0}$, and every $x \in Q_{0}$,

$$
\omega_{\lambda}\left(H_{\tau} f, Q_{0}\right) \leq C_{\tau, n, \lambda} f_{Q_{0}^{\tau}}|f(x)| d x
$$




$$
M_{\lambda, Q_{0}}^{\#, d}\left(H_{\tau} f\right)(x) \leq C_{\tau, n, \lambda} M^{d} f(x) .
$$

Proof. It suffices to prove the first inequality; the second follows immediately from definition of $M_{\lambda, Q_{0}}^{\#, d}$. Fix $Q_{0}$ and write $H_{\tau}$ as the sum of two operators:

$$
H_{\tau} f(x)=H_{\tau}\left(f \chi_{Q_{0}^{\tau}}\right)(x)+H_{\tau}\left(f \chi_{\mathbb{R}^{n} \backslash Q_{0}^{\tau}}\right)(x) .
$$

We claim the second term is constant for all $x \in Q_{0}$. Let $Q$ be any dyadic cube. Then the corresponding term in the sum defining $H_{\tau}\left(f \chi_{\mathbb{R}^{n} \backslash Q_{0}^{\tau}}\right)(x)$ is

$$
\sum_{\substack{Q^{\prime}, Q^{\prime \prime} \in \Delta(Q) \\ 2^{-\tau n}|Q| \leq\left|Q^{\prime}\right|,\left|Q^{\prime \prime}\right|}} a_{Q^{\prime}, Q^{\prime \prime}}\left\langle f \chi_{\mathbb{R}^{n} \backslash Q_{0}^{\tau}}, h_{Q^{\prime}}\right\rangle h_{Q^{\prime \prime}}(x) .
$$

We may assume that $Q^{\prime \prime} \cap Q_{0} \neq \varnothing$ (otherwise we get a zero term); since $Q^{\prime \prime} \subset Q$, this implies that $Q \cap Q_{0}^{\tau} \neq \varnothing$. Similarly, we have $Q \cap\left(\mathbb{R}^{n} \backslash Q_{0}^{\tau}\right) \neq \varnothing$. Therefore, $Q_{0}^{\tau} \subsetneq Q$, so $\left|Q_{0}\right|<2^{-\tau n}|Q| \leq\left|Q^{\prime \prime}\right|$. Hence, $Q_{0} \subsetneq Q^{\prime \prime}$ and $h_{Q^{\prime \prime}}$ is constant on $Q_{0}$. Thus, (6) does not depend on $x$ and so is constant on $Q_{0}$.

Denote this constant by $H_{\tau} f\left(Q_{0}\right)$; then

$\left|\left\{x \in Q_{0}:\left|H_{\tau} f(x)-H_{\tau} f\left(Q_{0}\right)\right|>t\right\}\right|=\left|\left\{x \in Q_{0}:\left|H_{\tau}\left(f \chi_{Q_{0}^{\tau}}\right)(x)\right|>t\right\}\right|$.

Since $H_{\tau}$ is a CZ Haar shift operator it is weak $(1,1)$. Therefore, by inequality (3),

$$
\begin{aligned}
\omega_{\lambda}\left(H_{\tau} f, Q_{0}\right) & \leq\left(\left(H_{\tau} f-H_{\tau} f\left(Q_{0}\right)\right) \chi_{Q_{0}}\right)^{*}\left(\lambda\left|Q_{0}\right|\right) \\
& \leq \lambda^{-1}\left\|H_{\tau}\left(f \chi_{Q_{0}^{\tau}}\right)\right\|_{L^{1, \infty}\left(Q_{0},\left|Q_{0}\right|^{-1} d x\right)} \leq \frac{C_{\tau, n}}{\lambda} f_{Q_{0}^{\tau}}|f(x)| d x .
\end{aligned}
$$

\section{The PRoOf of TheOREM 1}

Proof of Theorem 1. Fix $w \in A_{2}$ and fix $f$. By a standard approximation argument we may assume without loss of generality that $f$ is bounded and has compact support. Let $\mathbb{R}_{j}^{n}, 1 \leq j \leq 2^{n}$, denote the $n$-dimensional quadrants in $\mathbb{R}^{n}$ : that is, the sets $I^{ \pm} \times I^{ \pm} \times \cdots \times I^{ \pm}$ where $I^{+}=[0, \infty)$ and $I^{-}=(-\infty, 0)$.

For each $j, 1 \leq j \leq 2^{n}$, and for each $N>0$ let $Q_{N, j}$ be the dyadic cube adjacent to the origin of side length $2^{N}$ that is contained in $\mathbb{R}_{j}^{n}$. Since $Q_{N, j} \in \Delta, \Delta\left(Q_{N}\right) \subset \Delta$. Since $H^{\tau}$ is a $\mathrm{CZ}$ shift operator its adjoint is as well; thus, $H^{\tau}$ is bounded on $L^{p}, 1<p<\infty$. In particular, 
8 DAVID CRUZ-URIBE, SFO, JOSÉ MARÍA MARTELL, AND CARLOS PÉREZ

by (5) and (4), $m_{H_{\tau} f}\left(Q_{N, j}\right) \rightarrow 0$ as $N \rightarrow \infty$. Therefore, by Fatou's lemma and Minkowski's inequality,

$$
\left\|H_{\tau} f\right\|_{L^{2}(w)} \leq \liminf _{N \rightarrow \infty} \sum_{j=1}^{2^{n}}\left(\int_{Q_{N, j}}\left|H_{\tau} f(x)-m_{H_{\tau} f}\left(Q_{N, j}\right)\right|^{2} w(x) d x\right)^{1 / 2} .
$$

Hence, it will suffice to prove that each term in the sum on the right is bounded by $C_{\tau, n}[w]_{A_{2}}\|f\|_{L^{2}(w)}$.

Fix $j$ and let $Q_{N}=Q_{N, j}$. By Theorem 3 and Lemma 7, for every $x \in Q_{N}$ we have that

$$
\begin{aligned}
\mid H_{\tau} f(x)- & m_{H_{\tau} f}\left(Q_{N}\right) \mid \\
& \leq 4 M_{\frac{1}{4}, Q_{N}}^{\#, d}\left(H_{\tau} f\right)(x)+4 \sum_{j, k} \omega_{\frac{1}{2^{n+2}}}\left(H_{\tau} f, \widehat{Q}_{j}^{k}\right) \chi_{Q_{j}^{k}}(x) \\
& \leq C_{\tau, n} M f(x)+C_{\tau, n} \sum_{j, k}\left(f_{P_{j}^{k}}|f(x)| d x\right) \chi_{Q_{j}^{k}}(x) \\
& =C_{\tau, n} \operatorname{Mf}(x)+C_{\tau, n} F(x),
\end{aligned}
$$

where $P_{j}^{k}=\left(\widehat{Q}_{j}^{k}\right)^{\tau}$. We get the desired estimate for the first term from Buckley's theorem [2] with $p=2$ :

$$
\|M f\|_{L^{2}\left(Q_{N}, w\right)} \leq\|M f\|_{L^{2}(w)} \leq C_{n}[w]_{A_{2}}\|f\|_{L^{2}(w)} .
$$

To estimate $F$ we use duality. Fix a non-negative function $h \in L^{2}(w)$ with $\|h\|_{L^{2}(w)}=1$. We use the weighted dyadic maximal operator defined by

$$
M_{\sigma}^{d} g(x)=\sup _{x \in Q \in \Delta} \frac{1}{\sigma(Q)} \int_{Q}|f(x)| \sigma(x) d x .
$$

where $\sigma$ is a weight (i.e., locally integrable and positive a.e.). In particular we use that $M_{\sigma}^{d}$ is bounded on $L^{2}(\sigma)$ with constant bounded by 2 (see [11, Chapter 1, Exercise 1.3.3]). Therefore, by Remark 4 we have that

$$
\begin{aligned}
\int_{Q_{N}} F(x) h(x) w(x) d x & =C_{\tau, n} \sum_{j, k} f_{P_{j}^{k}}|f(x)| d x \int_{Q_{j}^{k}} w(x) h(x) d x \\
\leq 2 & \cdot 2^{(\tau+1) n} \sum_{j, k} \frac{w\left(P_{j}^{k}\right)}{\left|P_{j}^{k}\right|} \frac{w^{-1}\left(P_{j}^{k}\right)}{\left|P_{j}^{k}\right|}\left|E_{j}^{k}\right| \\
& \times \frac{1}{w^{-1}\left(P_{j}^{k}\right)} \int_{P_{j}^{k}}|f(x)| w(x) w(x)^{-1} d x
\end{aligned}
$$




$$
\begin{gathered}
\times \frac{1}{w\left(Q_{j}^{k}\right)} \int_{Q_{j}^{k}} h(x) w(x) d x \\
\leq C_{\tau, n}[w]_{A_{2}} \sum_{j, k} \int_{E_{j}^{k}} M_{w^{-1}}^{d}(f w)(x) M_{w}^{d}(h)(x) d x \\
\leq C_{\tau, n}[w]_{A_{2}} \int_{\mathbb{R}^{n}} M_{w^{-1}}^{d}(f w)(x) M_{w}^{d}(h)(x) d x \\
\leq C_{\tau, n}[w]_{A_{2}}\left(\int_{\mathbb{R}^{n}} M_{w^{-1}}^{d}(f w)(x)^{2} w(x)^{-1} d x\right)^{1 / 2} \\
\times\left(\int_{\mathbb{R}^{n}} M_{w}^{d}(h)(x)^{2} w(x) d x\right)^{1 / 2} \\
\leq C_{\tau, n}[w]_{A_{2}}\left(\int_{\mathbb{R}^{n}}|f(x) w(x)|^{2} w(x)^{-1} d x\right)^{1 / 2} \\
\quad \times\left(\int_{\mathbb{R}^{n}} h(x)^{2} w(x) d x\right)^{1 / 2} \\
=C_{\tau, n}[w]_{A_{2}}\left(\int_{\mathbb{R}^{n}}|f(x)|^{2} w(x) d x\right)^{1 / 2} .
\end{gathered}
$$

If we take the supremum over all such functions $h$, we conclude that

$$
\|F\|_{L^{2}\left(Q_{N}, w\right)} \leq C_{\tau, n}[w]_{A_{2}}\|f\|_{L^{2}(w)} .
$$

Combining our estimates we have that

$$
\left(\int_{Q_{N}}\left|H_{\tau} f(x)-m_{H_{\tau} f}\left(Q_{N}\right)\right|^{2} w(x) d x\right)^{1 / 2} \leq C_{\tau, n}[w]_{A_{2}}\|f\|_{L^{2}(w)},
$$

and this completes the proof.

\section{REFERENCES}

[1] K. Astala, T. Iwaniec, and E. Saksman. Beltrami operators in the plane. Duke Math. J., 107(1):27-56, 2001.

[2] S. M. Buckley. Estimates for operator norms on weighted spaces and reverse Jensen inequalities. Trans. Amer. Math. Soc., 340(1):253-272, 1993.

[3] L. Carleson. Two remarks on $H^{1}$ and BMO. Advances in Math., 22(3):269-277, 1976.

[4] D. Cruz-Uribe, J. M. Martell, and C. Pérez. Sharp two-weight inequalities for singular integrals, with applications to the Hilbert transform and the Sarason conjecture. Adv. Math., 216(2):647-676, 2007.

[5] D. Cruz-Uribe, J. M. Martell, and C. Pérez. Sharp weighted estimates for classical operators. Preprint, 2010. 
10 DAVID CRUZ-URIBE, SFO, JOSÉ MARÍA MARTELL, AND CARLOS PÉREZ

[6] O. Dragičević, L. Grafakos, M. Pereyra, and S. Petermichl. Extrapolation and sharp norm estimates for classical operators on weighted Lebesgue spaces. Publ. Mat., 49(1):73-91, 2005.

[7] J. Duoandikoetxea. Fourier analysis, volume 29 of Graduate Studies in Mathematics. American Mathematical Society, Providence, RI, 2001. Translated and revised from the 1995 Spanish original by D. Cruz-Uribe.

[8] N. Fujii. A proof of the Fefferman-Stein-Strömberg inequality for the sharp maximal functions. Proc. Amer. Math. Soc., 106(2):371-377, 1989.

[9] N. Fujii. A condition for a two-weight norm inequality for singular integral operators. Studia Math., 98(3):175-190, 1991.

[10] J.B. Garnett and P.W. Jones. BMO from dyadic BMO. Pacific J. Math., 99(2):351-371, 1982.

[11] L. Grafakos. Classical Fourier analysis, volume 249 of Graduate Texts in Mathematics. Springer, New York, second edition, 2008.

[12] R. Hunt, B. Muckenhoupt, and R. Wheeden. Weighted norm inequalities for the conjugate function and Hilbert transform. Trans. Amer. Math. Soc., 176:227-251, 1973.

[13] B. Jawerth and A. Torchinsky. Local sharp maximal functions. J. Approx. Theory, 43(3):231-270, 1985.

[14] M. Lacey, S. Petermichl, and M. Reguera-Rodriguez. Sharp $A_{2}$ inequality for Haar shift operators. Preprint, 2009.

[15] A. Lerner. A pointwise estimate for local sharp maximal function with applications to singular integrals. Preprint, 2009.

[16] F. Nazarov, S. Treil, and A. Volberg. Two weight inequalities for individual Haar multipliers and other well localized operators. Math. Res. Lett., 15(3):583-597, 2008.

[17] S. Petermichl. Dyadic shifts and a logarithmic estimate for Hankel operators with matrix symbol. C. R. Acad. Sci. Paris Sér. I Math., 330(6):455-460, 2000.

[18] S. Petermichl. The sharp bound for the Hilbert transform on weighted Lebesgue spaces in terms of the classical $A_{p}$ characteristic. Amer. J. Math., 129(5):1355-1375, 2007.

[19] S. Petermichl. The sharp weighted bound for the Riesz transforms. Proc. Amer. Math. Soc., 136(4):1237-1249, 2008.

[20] S. Petermichl and A. Volberg. Heating of the Ahlfors-Beurling operator: weakly quasiregular maps on the plane are quasiregular. Duke Math. J., 112(2):281-305, 2002.

David Cruz-Uribe, SFO, Dept. of Mathematics, Trinity College, HARTFORD, CT 06106-3100, USA

E-mail address: david.cruzuribe@trincoll.edu

José María Martell, Instituto de Ciencias Matemáticas CSiC-UAMUC3M-UCM, Consejo Superior de Investigaciones Científicas, C/ SerRANO 121, E-28006 MADRID, SPAIN

E-mail address: chema.martell@uam.es

Carlos PÉrez, Departamento de Análisis Matemático, Facultad de Matemáticas, Universidad de Sevilla, 41080 Sevilla, Spain

E-mail address: carlosperez@us.es 